\title{
Multi-modal Sensing Techniques for Interfacing Hand Prostheses: a Review
}

\author{
Yinfeng Fang, Nalinda Hettiarachchi, Dalin Zhou, and Honghai Liu
}

\begin{abstract}
This paper provides a comprehensive survey of current state of the bio-sensing technologies focusing on hand motion capturing and its application to interfacing hand prostheses. These sensing techniques include electromyography (EMG), sonomyography (SMG), mechnomyography (MMG), electroneurography (ENG), electroencephalograhy (EEG), electrocorticography (ECoG), intracortical neural interfaces, near infrared spectroscopy (NIRS), magnetoencephalography (MEG) and functional magnetic resonance imaging (fMRI), etc. Relevant approaches that interpret bio-signals in the view of prosthetic hand manipulation are involved in as well. Multi-modal sensory fusion provides a new strategy in this area, and the latest multi-modal sensing techniques are surveyed. The paper also outlines the new challenges and directions: exploration of robust sensing technology, multi-modal sensory fusion, on-line signal processing and learning algorithms and bio-feedbacks.
\end{abstract}

Keywords-bio-sensing technology, electromyography, sonomyography, mechnomyography, electroencephalograhy, hand prostheses, multi-modal sensing.

\section{INTRODUCTION}

$\mathbf{F}$ UNCTIONALITY, controllability and cosmetics are the key issues to be addressed in order to accomplish a successful functional substitution of the human hand by means of a prosthesis [1]. To implement the controllability, bodysensing technologies should be applied to collect bio-signals that are related to native hand motions. Then the signals should be transformed into control commands to artificial hands in the most intuitive and undemanding way.

Current commercial prosthetic hands generally fall into two groups: passive/cosmetic and active/functional prosthetic hands. A cosmetic prosthetic hand mimics the shape of a hand without manipulation ability. An active hand prosthesis can be voluntarily actuated by the patient wearing it [2], which can be either body-powered or electrically powered [3]. To successfully manipulate a prosthetic hand, patients must be able to exert the correct grasping type with proper grasping force, and meanwhile to receive a perceptional feedback of manipulations. Traditional input devices, like slider switches and force-sensing resistors [4], can guarantee the working stability, but end in a humdrum and non-intuitive control.

As prosthetic hands of multiple degrees of freedom (DoFs) with high dexterity come to fruition, it will be necessary for the control systems to follow suit [5]. Although modern multifunctional prosthetic hands, such as I-Limb (Touch

The authors are with Intelligent Systems and Biomedical Robotics Group, School of Computing, University of Portsmouth, UK e-mail: \{yinfeng.fang;nalinda.hettiarachchi;dalin.zhou;honghai.liu\}@ port.ac.uk. Corresponding author: H. Liu.
Bionics Inc., UK), have the provision of controlling individual fingers, user interfaces can hardly control individual fingers [6]. The interface between users and machines becoms the bottle-neck, which explains why current hand prostheses are functionally plain from a biomechanic point of view [1]. To ensure a successful clinical evaluation and commercial exploitation, prosthetic hands are required to possess a robust, reliable and intuitive interface supporting dexterous control [7], [8]. Besides, diverse bio-signals obtained through a variety of human machine interfaces (HMIs) should be employed easily by the prostheses.

Generally, HMIs can be categorised into invasive and noninvasive interfaces. Invasive approaches usually need surgery to implant electrodes in the human body, such as ECoG, peripheral nervous interfaces (PNIs), whereas non-invasive interfaces connect patients and prostheses via fixing sensing electrodes on the surface of the skin, such as surface/superficial EMG (sEMG), EEG, MMG, etc. Invasive interfaces can deliver bio-signals with higher quality, while non-invasive interfaces can be easily handled and maintained physically, but requires preciser follow-on signal conditioning methods. In either the invasive or the non-invasive interface, a variety of body sensing techniques have been employed to provide diverse bio-signals to achieve dexterous control of artificial hands. Recently, various multi-modal sensing techniques have been developed like the combinations of EMG and accelerometers, MMG and accelerometers, and EEG and ENG. Literatures revealed that multi-sensing technology can interpret hand motions with higher accuracy than uni-modal bio-signals.

The remaining of this review is organised as follows: Section II investigates the EMG and some relevant topics in prosthetic hand interaction; Sections III and IV survey state-of-theart SMG and MMG, respectively; Section V demonstrates PNIs and the corresponding ENG; Section VI discusses the functionalities of a variety of HMIs such as EEG, ECoG, MEG and fMRI; Section VII presents multi-sensory fusion strategies in improving hand motion classification performance; Section VIII proposes some challenges and directions; the paper is concluded in the last section.

\section{ELECTROMYOGRAPHY}

EMG is a technique used for evaluating and recording the activation signal of muscles and also utilized for the electrical manifestation of the contractions of muscles [9], [10]. Remaining muscles in an amputation stump hide sleeping potentials for the generation of specific patterns of EMG signals corresponding to complex hand movements [8], underlying any myoelectric prosthesis. 
EMG is divided into two main groups: needle/intramuscular/internal EMG and sEMG. Needle EMG is of high selectivity and less representativity of the global muscle activity. To obtain needle EMG signals, electrodes need to be inserted into the muscle tissue through skin. Therefore, few studies apply it for prosthetic hand interaction due to the invasiveness [11]. However, it provides possibility of chronic implants [12]. For instance, Pulliam, etc., [3] integrated a fully implanted EMG recording system with a wireless telemetry system that communicates with prostheses. sEMG, a technique by which muscle activation potentials are gathered by electrodes placed on patients' skin; these potentials can be used to track which muscles the patient is willing to activate and determine what force is to be adopted [2]. Although needle EMG has less muscular crosstalk allowing for more independent control sites, there is no significant difference in terms of accuracy in discriminating wrist and grip movements [13]. Hence, sEMG has attracted remarkable attention in the design and manufacturing of artificial limbs[14].

As far as myoelectric control systems are concerned, the quality of signals should be first taken into account. The EMG signal is non-stationary [15] and easy to be contaminated by a wide variety of factors, like ambient noises, motion artefacts, the physiological and anatomical properties of a patient [16]. To deal with it, Hargrove, etc., [13] proposed two major approaches to increase the accuracy of prosthetic controllers: 1) use signal processing to extract more information from the input signals; 2) provide more informative raw signals to the controller.

\section{A. sEMG Electrode Configuration}

sEMG signals is most commonly collected using one or more electrodes placed on the skin surface either with reference to particular muscles or equidistantly over an area of interest [17]. sEMG can be captured by wet/dry electrodes [18]. sEMG electrodes can be in passive or active mode. However, active electrodes can not provide higher hand motion classification accuracy as expected [19]. Recently, Li etc., [18] proposed a kind of textile electrode fabricated with copper-based nickel-plated conductive fabric. And this kind of electrode can reach a similar performance as the gelled metal electrode in the task of classifying arm movements.

The importance of electrode configuration lies on its control accuracy, prosthetic production cost, computational load, etc. The layout of surface electrodes can be classified as: 1) Muscle-targeted layout, requiring to pinpoint muscles and adhere pairs of electrodes on the belly, as demonstrated in [2], [20], [21]. 2) Low-density surface electrode layout, arranging electrodes in certain patterns and distributed evenly on the skin forming ring or belt structures, which is also known as uniform electrode positioning strategy [22]. The channel number of this configuration usually varies from two to sixteen. The concept of low-density sEMG was first introduced by Huang etc., [23], where six bipolar electrodes were distributed uniformly at $1 / 3$ of the distance from elbow to wrist. 3) High-density (HD) surface electrode layout, collecting EMG signals from closely spaced electrodes [24], which would allow to exploit the spatial information across muscles. Therefore such strategy can be more useful for the study of complex dynamic tasks in the free space and with a greater number of DoFs [25]. HD-EMG is usually related to energy maps that illustrate what electrode specifications experience strong myoelectric activity during diverse motion tasks. These maps can also be examined to determine whether distinguishable muscle activation patterns are produced [26]. However, it remains a challenge to deal with a large number of EMG channels for interacting a prosthetic hand in practice.

The controversy towards optimal electrode number for EMG signal collection for prosthetic hand control can be identified between industry and literatures. For most commercial myoprosthetic hands, two electrode pairs on the extensor and the flexor respectively are usually adopted due to space limitations [27], power consumption [25], real-time performance [28], etc. It is possible to provide natural, reliable myoelectric control via increasing EMG channels across multiple muscles, but add to the complexity of subsequent processing and analysis. On the one hand, researchers tend to carry out experiments with multiple electrodes. A reduction in the electrode number without compromising the accuracy has attracted great attention, by which the requirements for controlling prostheses would be significantly simplified [29]. Young, etc., [30] and $\mathrm{Li}$, etc. [31] presented that four to six channels were sufficient for pattern recognition (PR) based prosthetic manipulation. In addition, Huang, etc., [32] demonstrated that 12 selected bipolar electrodes can obtain a similar classification accuracy (only $1.2 \%$ drops) compared with the entire HD electrode layout in a targeted muscle reinnervation (TMR) application. Literatures focusing on prosthetic hand control using different amounts of EMG electrodes can be found in Table I.

It is abrupt to justify the pros and cons of electrode configurations by simply comparing the classification accuracy of different studies, because the diversity of numbers of classification movements, the type and durations of movements, the utilised classification systems, the number of subjects and the data set sizes all contribute to various performance [17]. However, the proposed methodologies have reference values, from which we conclude two points on the electrode configuration that may promote further studies in both academics and industries. Firstly, to extract discriminable patterns from EMG signals, precise placement of electrodes on specific muscles is not necessary [33], and low-density electrode layout can attain the same target as tested in [22]. Secondly, in terms of hand motion classification, a limited number of electrodes can reach an acceptable classification accuracy as using more electrodes. However, the influence of compromising in electrode number varies with different electrode layout as revealed in [17], [23], [25], [26], [34].

\section{B. Pattern Recognition based Prosthetic Control}

To implement myoelectric prosthetic control, several approaches can be utilised including on/off control, proportional control, PR based control and finite-state-machine control. A comprehensive review of terminology and proportional myoelectric control was publised by Fougner, etc. [35]. Commercial systems allowing to control over more than one DoFs tend 
to exploit finite-state-machine controllers [36]. This review concentrates on PR based prosthetic control methods.

PR based control offers means of extracting more information from available muscles than other conventional methods. Therefore, it is possible to provide more natural, reliable control for myoelectric prostheses [26]. PR myoelectric control is generally divided into four phases: data segmentation, feature extraction, classification and controller, as described in [37].

Although great efforts have been made on PR based prosthetic control, few clinically viable systems have been implemented with this strategy [38]. Recent literature has highlighted a disparity between classification accuracy and usability on PR based prostheses [39]. To narrow this gap, Scheme, etc., [39] introduced a selective multi-class oneversus-one classification scheme, capable of rejecting unknown data patterns, while Chen, etc., [40] proposed a self-enhancing classification method that incorporated the knowledge beyond the training condition to the classifiers from the testing data. A number of recent studies aiming to improve EMG based prosthetic hand control can be found in Table I.

\section{On-line Training}

PR based prosthetic hand has given priority to off-line analysis through real-time processing and classification algorithms, as presented in Table I. Only a few studies involve in real-time EMG PR scheme for prosthetic hand control.

In clinical applications, characteristics of the collected sEMG signals vary with time, and make every PR based control system face exponentially rising error over long-time operation [16], [37], [47], [48]. Therefore, whether the offline trained classifier can be successfully applied to real applications is still in question. The possible factors causing the above phenomenon are: electrode displacement, skin impedance changes, artefacts, prosthetic donning/doffing, and separation of intention from other physical factors such as fatigue, stump posture, etc.

Online training [37], where a classifier is trained continuously using new patterns during operation, makes the rate of accuracy stable and insensitive to long-term operation. Khezri, etc. proposed a real-time scheme for hand prosthetic control, as shown in Fig. 1, in which an online training unit is utilised to link actual EMG patterns with generated control commands and further adapt to the operators' characteristics [42]. Castellini, etc. presented a simple but effective procedure for selecting a subset of the samples on-the-fly, called online uniformisation, which is effective in building a compact and accurate training set for support vector machine (SVM)[2]. Amsuss, etc. developed a self-correcting PR system to improve the classification accuracy, through which the erroneous classification decisions are pretentiously detected[38]. Pilarski, etc. claimed that they proposed the first demonstration of a practical method for real-time prediction learning during myoelectric interacting with a prosthesis[53].

\section{Simultaneous Movements}

PR based myoelectric control systems employ a sequential strategy, where only one function of the prosthetic device can

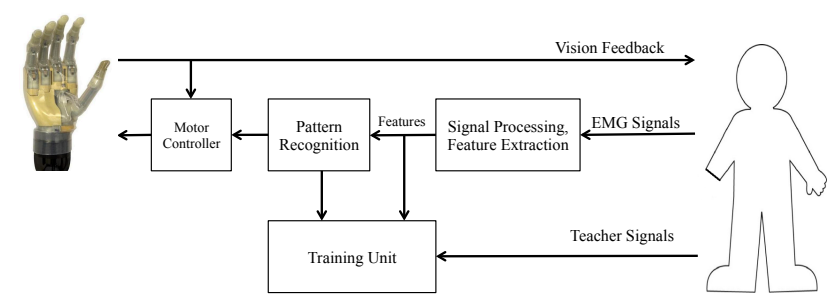

Fig. 1. Real-time scheme for hand prosthesis control, modified from [42]

be activated at a time, and sequential control of each DoF is slow and tedious [54]. Conversely, natural limb movements consist in the continuous and simultaneous activation of multiple DoFs. The continuous prediction of trajectories has the potential advantage of allowing coordinated and simultaneous control of multiple DoFs in a natural manner [3]. Therefore, except for classification to assign a label to each sample in the input space, regression can be applied to get the realvalues of input samples [2]. A comparison report on various linear and nonlinear regression techniques for simultaneous and proportional myoelectric control of wrist movements is demonstrated in [55]. More issues regarding mapping EMG signals into simultaneous and proportional control signals are discussed in [36].

From another perspective, multiple DoF activation needs to selectively modulate EMG signals in order to make each joint move independently, and this evidently requires significant cognitive effort to users [1]. Recent researches resort to muscle synergy frameworks that systematically regards several joints as an entirety, and all DoFs of a dexterous robotic hand may be controlled by several synergies, which reduces users' burden in attention. Moreover, synergy is able to predict the EMG patterns associated with untrained static hand postures [56].

\section{E. Able/Disable Subjects}

Since practical and ethical issues, only a few researches are able to employ amputees to implement their experiments. Any study based on healthy subjects is under a hypothesis that the remaining muscles in the forearm are still sufficient to generate efficient EMG signals for prosthetic control [21]. Although some studies report that there is not much difference in motion decoding accuracy between amputee and able-bodied subjects [5], [7], [23], it is still a challenge to implement multifunctional myo-prosthetic manipulation in clinic. Firstly, amputees are required to wear a prosthetic socket over the day. EMG signals not only contribute to the intended hand motions, but also to sustain the weight of a prosthesis [57]. Therefore, the corresponding EMG signal collected on amputees with prosthetic sockets may not completely represent amputee's intentions. Cipriani, etc., [7] disclose that a sEMG pattern caused by a simple lifting or moving of the prosthesis can be misclassified into a hand control movement. Secondly, muscular differences caused by different surgical procedures lead to a unique case that requires individualised attention [26]. Thus, special treatment for amputees with different amputation levels is required [5]. Thirdly, prosthetic users may differ in 
TABLE I. RESEARCHES ON EMG-BASED HAND MOTION CLASSIFICATION

\begin{tabular}{|c|c|c|c|c|c|c|c|c|}
\hline Paper & Electrodes & Motions & Segmentation(ms) & Feature $^{8}$ & Classifier & $\operatorname{Accuracy}(\%)$ & Real-time & Note * \\
\hline [6] & $1^{1} / \mathrm{MT}^{2}$ & 4 & $1000^{5} / 0^{6}$ & FD+MEL $^{9}$ & ANN & 90.7 & no & \\
\hline [41] & 2/MT & 10 & $187.5 / 31.3$ & $13 \mathrm{TD}^{10}, \mathrm{FD}+$ OFNDA & SVM+MLP & 87.8 & considered & \\
\hline [42] & $2 / \mathrm{MT}$ & 6 & $200 / 50^{*}$ & $2 \mathrm{TD}+1 \mathrm{TFR}$ & ANFIS & 96.7 & yes & $50 \mathrm{~ms}$ subwindow \\
\hline [21] & $2 / \mathrm{MT}$ & 6 & & DWT & SVM & 97.5 & no & \\
\hline [9] & $2 / \mathrm{MT}$ & 8 & $200 / 50^{*}$ & 6TD,STFR,WT+PCA & FIS & 83.3 & no & $50 \mathrm{~ms}$ subwindow \\
\hline [21] & 2/MT & $6^{*}$ & $\mathrm{NM}^{7}$ & $\mathrm{DWT}+\mathrm{PCA}$ & SVM & 97.5 & no & Grasp motions \\
\hline [43] & $2 / \mathrm{MT}$ & 4 & 200/NM & $1 \mathrm{TD}$ & C-Means & $92.7 \pm 3.3$ & yes & \\
\hline [44] & 4/MT & 6 & $1000 / \mathrm{NM}$ & RoughEn & SVM & $95.2 \pm 3.0$ & no & \\
\hline [20] & 4/MT & 7 & NM & $8 \mathrm{TD}, 2 \mathrm{FD}$ & ANN & 92.0 & considered & \\
\hline [45] & 4/MT & 5 & $256 / 128$ & Bispectrum & ANN & 92 & yes & \\
\hline [46] & 4/MT & 6 & $256 / 128$ & $4 \mathrm{TD}$ & SLR +DT* & $91 \pm 1.9$ & no & Low computing and memory cost \\
\hline [23] & $6 / \mathrm{LD}^{3}$ & 8 & $100 / \mathrm{NM}$ & CSSP & LDA & $98 / 80.3^{*}$ & no & Able-bodied subjects/Amputees \\
\hline [47] & 8/LD & 9 & $200 / 175$ & $3 \mathrm{TD}$ & LDA & $77.5 / 94.4^{*}$ & yes & Before/after systematic training \\
\hline [48] & 6/MT & $\begin{array}{l}9 \\
18\end{array}$ & NM & NM & Thd+SVM & $\begin{array}{l}85.9 \pm 8.34 \\
73.5 \pm 8.3\end{array}$ & yes & \\
\hline [22] & 6/LD & $5+1^{4}$ & NM & NM & SVM & $95^{*}$ & no & Three amputees \\
\hline [49] & $7 / \mathrm{LD}^{*}$ & 11 & NM & $6 \mathrm{TD}$ & GRA & 95.9 & considered & Active electrode \\
\hline$[50],[51]$ & 8/MT & $6+1$ & $256 / \mathrm{NM}$ & $6 \mathrm{TD}, \mathrm{FR} *+\mathrm{DEFLDA}$ & LDA & 94.7 & considered & \\
\hline [7] & $8 / \mathrm{MT}$ & 7 & $250 / 50$ & MAV & KNN & $89 / 79^{*}$ & yes & Able-bodied subjects/Amputees \\
\hline [2] & $10 / \mathrm{LD}$ & 4 & NM & $\mathbf{O U}$ & SVM & 92.6 & yes & \\
\hline [52] & $8 \mathrm{LD}+4 \mathrm{MT}$ & $10+1$ & $150 / 50$ & 4TD & LDA & $84.4 \pm 7.2$ & yes & \\
\hline [29] & 32/LD & $12^{*}$ & $200 / 25$ & $4 \mathrm{TD}$ & ANN & $>98$ & no & Towards finger motions \\
\hline [5] & $\begin{array}{l}\text { 32/LD } \\
19 / \mathrm{LD}\end{array}$ & 6 & $200 / 25$ & $4 \mathrm{TD}^{*}$ & $\mathrm{NN}$ & $\sim 90$ & considered & \\
\hline
\end{tabular}

${ }^{1}$ EMG channel number; ${ }^{2}$ Muscle-targeted electrode configuration; ${ }^{3}$ Low density electrode configuration;

${ }^{4}$ Several hand motions and one rest motion; ${ }^{5}$ Window length; ${ }^{6}$ Incremental window length; ${ }^{7}$ Not mentioned; ${ }^{8}$ Features and dimension reduction methods;

${ }^{9}$ Bold character indicates it is the key contribution of the study; ${ }^{10} 13$ TD features.

learning capacity, and appropriate type of control for each patient should be taken into account [58]. For example, there exits obvious difference between unimpaired participants and cosmetic prostheses users in the process of training [7].

\section{F. Targeted Muscle Reinnervation}

In 2004, Kuiken, etc., [59] present a surgical technique called TMR transfers residual arm nerves to alternative muscle sites. Once reinnervated, these muscles serve as biological amplifiers of motor commands from the transferred arm nerves and provide physiologically appropriate EMG signals for the control of the prosthetic elbow, wrist, and hand. EMG based prosthetic hand control after TMR supply several advantages of intuitiveness, high level amputation satisfaction, sensory feedback, and efficient simultaneous multi-DoFs control [60].

\section{SONOMYOGRAPHY}

Ultrasound (US) imaging, also known as medical ultrasonography is a noninvasive technique to visualise structures inside the human body. The use of US imaging to exam target muscle contraction is termed sonomyography [61]. For several decades, US imaging techniques have been successfully used as a diagnostic tool for forearm musculoskeletal disorders [62]. Recently, researchers start to employ US as an HMI to interact external devices, particularly for prosthetic control. A human hand is controlled by both the superficial and deep muscles on the dorsal and ventral sides of the forearm, and therefore detecting deeper muscular activity is necessary. Compared with sEMG which is unable to detect deep muscle activities, US remedies this defect. Besides, US also overcomes the inherent weakness of electromagnetic bio-signals, like EMG, EEG, etc., all of which can be easily contaminated by electronic interference, like power-line noises.

In SMG, the interpretation and translation of morphological changes of forearm muscles are pivoted in interacting robotic prostheses, which has attracted great attention by researchers. When a finger/wrist flexes or extends, corresponding muscle thicknesses change. Fukumoto, etc., reveal that US is able to successfully identify muscle volume changes and estimate muscular strength [63]. Castellini, etc., [61], claim that there is a clear linear relationship between the features extracted from US images, and finger positions. Zheng, etc., [64] demonstrate that the selected echo features of US images can be employed to establish the relationship between wrist extension angle and the percentage of muscle deformation.

Furthermore, US has been used to predict and recognise simple wrist and finger movements. In [65], the joint angle of the wrist has been evaluated by tracking features in a window of US images. Zarka, etc., [66] conducted a notable research by extracting feature from US images to predict finger movements. Shi, etc., [67] utilise Horn-Schunk optical flow algorithm to identify five-finger flexions from US images, and have qualitatively proved that the directions of optical flow field and deformations areas of muscle contraction were diverse with different fingers' flexions. Sikdar, etc., [68] show the success of individual finger movement classification at $98 \%$ accuracy using a standard ultrasound probe.

All the above studies are based on the well-known blackwhite mode (B-mode) US, where image processing technologies are usually required to better understand US images. A few studies also involve amplitude mode (A-mode) US [69] and time-of-flight US [70]. By utilising A-mode US, Change, etc., [69] have clearly highlighted a linear relationship of muscular contraction and A-mode 1D single element US 
feature variations, contrary to the non-linear relationship of sEMG. Tsutsui, ect., [70] show that it is possible to recognise human joint motion using ultrasound pulse echoes.

\section{MECHNOMYOGRAPHY}

MMG is a non-invasive muscle activity detection method, and also known as vibromyography and acoustic-myogram (phono-myography). It measures and quantifies lateral oscillations generated by dimensional changes in active muscle fibres [71] at frequencies ranging from 5 to $100 \mathrm{~Hz}$. These signals can be detected by microphones or low mass accelerometers [72]. They have been studied for pain monitoring, tracking of muscle fatigue, measurement of muscle contractility in myopathic diseases, and bi-functional prosthetic upper-limb control [73].

MMG has been employed to detect forearm flexions and extensions and the exerted force. Lei, etc., [74] find that the root mean square (RMS) of MMG signals increased monotonously and the frequency variance decreased under incremental voluntary contraction. Cole, etc., [72] used two wavelet components of MMG signals to predict the isometric contraction force of brachioradialis muscle, and their results show that MMG is better in estimating the force rather than gestures as vibratory signals are prominent with increasing force. $\mathrm{Ni}$, etc., [71] collected sEMG, MMG and US images simultaneously to investigate features during voluntary isometric ramp contraction of rectus femoris muscle, and the results show that MMG and EMG have a close relation with torque. It is worth to note that MMG has the potential to detect weaker contractions than EMG [75].

MMG has also been used in hand motion recognition. A study conducted by Zeng, etc., [76] shows that the statistical classification accuracy rate of $79.66 \% \pm 7.32 \%$ can be achieved in recognising four hand motion patterns using single MMG transducer fixed to upper arm, where 11 original features of MMG were extracted, principle components analysis was employed to reduce feature dimension, and quadratic discriminant analysis was utilised for recognition.

\section{ELECTRONEUROGRAPHY}

Different form TMR approach that reconnects peripheral nerves to other less functional muscles, PNIs directly capture bio-signals from peripheral nervous system (PNS) via implanting electrodes, such as longitudinal intra-fascicular electrodes (LIFEs) [77], self-opening intrafascicular neural interfaces (SELINEs) [78], and the corresponding technique is termed ENG. Clinical peripheral nerve electrodes can be classified into three groups: intraneural, extraneural and miscellaneous, as discussed in [79], [80]. The use of invasive neural interfaces that directly connects to the PNS is potentially appealing because it is able to provide an almost "physio-logical" condition in which efferent and afferent fibres, previously liking to natural hands, may return to their role in interacting the prosthetic limb/hand [77]. Both the extraction of motor information and the restoration of sensory function are possible [81]. A review of neural machine interfaces for controlling multifunctional powered upper-limb prostheses can be referred to [79].
To implement the task of artificial hand manipulation by amputees, classical approaches are based on EMG signals, while the emergent interest is to use neural signals directly [82]. In 2005, Dhillon and Horch [83] firstly demonstrate the direct neural feedback and direct neural control of an artificial arm by amputees. Their approach allows amputees to judge and set grip force and joint positions of an artificial arm. Dhillon, etc. [84] discover that amputees were able to improve volitional control of motor neurone activity. In 2010, Rossini, etc., [85] achieved real-time control of motor output for three actions using multiple electrodes in different nerves with numerous contacts, and the correct classification reached to more than $85 \%$. Recent development by Micera, etc., [77] demonstrates that motor information, like grip types and single finger movements, could be extracted from PNIs and users could improve their ability to govern motor commands over time. Carpaneto, etc., [78] achieved classification accuracies of more than $85 \%$ using the best combination of ENG channels, and these accuracies are even comparable with EMG based classification.

\section{BRAIN-MACHINE INTERFACE}

A brain-machine interface (BMI), or brain-computer interface (BCI), can be defined as any system able to monitor brain activity and translate a person's intentions into commands to external devices in a non-muscular communication approach, instead of being sent to the physiological musculo-skeletal effectors [86], [87], [88]. It benefits patients who lost voluntary muscle contraction whereas the sensory, emotional and cognitive processing remain largely intact [88]. BMIs can be either invasive or non-invasive. For invasive BMIs, there are three types, ECoG, the local field potential (LFP) and spikes [89]. ECoG signals are measured over cortex, and electrodes were not inserted into cortex, which is different from LEP and spikes. Non-invasive recordings are obtained as electrical or magnetic activity from the scalp [88] via EEG, MEG, etc. Compared with invasive methods, non-invasive BMIs avoid the risks of brain surgery [90], but these signals are of low spatial resolution and susceptible to artefacts from other sources [91].

\section{A. Electroencephalograhy}

EEG is the most popular BMI because it is inexpensive and mobile [92]. However, it remains a challenge to achieve robust prosthetic control by EEG signals, and most literatures are still on hand motion discrimination. Harshavardhan, etc., [93] proposed an approach to design EEG-based decoders that can reconstruct finger and thumb joint angles during a reach-to grasp task with $76 \%$ accuracy from EEG signals. Mohamed, etc., [94] show that the average accuracies of $65 \%$ and $71 \%$ respectively with 5 executed and imagined movements can be achieved by EEG signal analysis. Bradberry, etc., [95] successfully decoded hand position, velocity, and acceleration from 55-channel EEG signals in three-dimensional center-out reaching tasks.

EEG signals are usually captured from a great number of channels. To make EEG based prosthetic hand manipulation practical, channel reduction can be adopted. Through selecting 
6 of 32 channels using a genetic neural mathematic method, Yang, etc., [90] achieved a classification accuracy of about $86 \%$ during the tasks of response to given motions. This result is higher than that of using entire channels, which is possible because that the EEG channels with irrelevant/noisy data are eliminated.

\section{B. Electrocorticography}

ECoG places electrodes directly on the exposed surface of the cerebral cortex to record electrical activity, seen in Fig. 2. Compared with intracortical electrodes, it takes advantages of lower clinical risk, greater long-term stability; compared with EEG, it can obtain higher spatial resolution, bandwidth [96], singal-to-noise ratio [91] and less attenuation in higher spectrum [86]. As such, among the possible cortical signals, ECoG offers one of the most clinically feasible options [97]. More importantly, ECoG can present an advantage on finger movements, since finger representations are more spread out in the cortex [98]. To drive different prosthetic movements, the existing signals in the motor cortex that are already associated with finger movements and grasps can be utilised, and ECoG signals that indirectly drive hand motions, like tongue movements, can be modulated as well [98]. But the latter is not able to satisfy the requirement of intuitiveness.

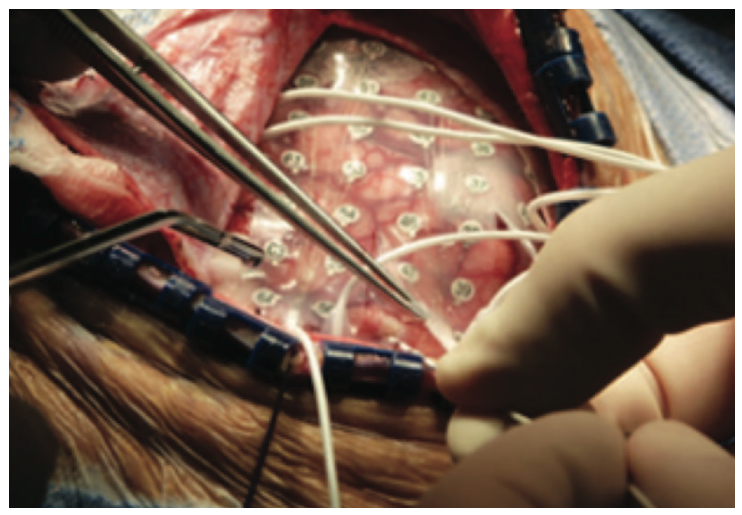

Fig. 2. The clinical surgery of ECoG electrode array implant [96]

Pioneers who pursue to decode hand or finger motions by ECoG have shown great confidence in interacting prosthetic hands. Chestek, etc., [98] have achieved classification accuracy at $68 \%, 84 \%$ and $81 \%$ for identifying 5 isometric hand postures offline from three participants who were undergoing intractable epilepsy. Their online experiment on fist versus rest classification reached up to 97\%. Yanagisawa, etc., [91], [97] used the power modulations of ECoG signals to control a prosthetic hand, and demonstrate the successful control of a prosthetic arm by the patients with motor dysfunctions. To achieve real-time ECoG control with high accuracy, Benz, etc., [99] used time-varying dynamic Bayesian networks to determine connectivity between ECoG channels in humans during motor tasks. Moreover, Fifer, etc., [96] also demonstrate that complex movements can be decoded from a patient's $\mathrm{ECoG}$ signal. Five future directions on $\mathrm{ECoG}$ control are concluded by Fifer, etc., [96]: improving the resolution of ECoG arrays, maturation of decoding algorithms, provision of proprioceptive and touch feedback, fully implanted ECoG systems and ethical considerations.

\section{Intracortical Neural Signals}

Intracortical neural signals being collected within the cortex by invasive electrodes are capable of interfacing hand prostheses. A type of intracortical microelectrode array (4 $\mathrm{mm} \times 4 \mathrm{~mm}$, Blackrock Microsystems, Salt Lake City, UT, USA) has been used to record neural signals in [100], [87], [101]. Hochberg, etc., [100] recorded neuronal ensemble activity in primary motor cortex and demonstrated that intended hand motion modulates cortical spiking patterns three years after spinal cord injury. Collinger, etc., [87] implanted two Blackrock arrays in the participant's left motor cortex, and after a period of training, the participant was able to use a prosthetic limb to do skilful and coordinated reach and grasp movements. Hochberg, etc.,[101] demonstrate people with long-standing tetraplegia are able to use intracortical neural signals to perform three-dimensional reach and grasp movements without explicit training.

\section{Other BMIs}

Other technologies recording brain activities include MEG measuring neuromagnetic signals, fMRI testing blood oxygen level-dependent responses and NIRS evaluating the activityrelated brain oxygenation [91]. Several studies based on the above technologies have been completed to demonstrate their potentials as a BMI to interact prosthetic hand via decoding signal patterns of hand motions.

Sugate, etc., [102] used MEG signals to classify three types of right upper limb movements: grasping, pinching and elbow flexion, and obtained an average accuracy at $66 \pm 10 \%$. Kauhanen, etc., [103] utilised MEG signals to classify the motions of lifting the left, right or both index fingers, and achieved accuracies from $80 \%$ to $94 \%$ (two-category) and from $57 \%$ to 67\% (three-category). Quant, etc.,[104] find that the MEG decoding accuracy is more robust than EEG's performance during a task of classifying finger motions by pressing a single button with different fingers. However, another study conducted by Kauhanen, etc., [92] show that MEG and EEG have the similar performance in classifying three index finger movements.

Maruishi, etc., [105] used fMRI to localise activation in the human brain during the manipulation of a prosthetic hand and find that the robotic hand might be recognised in the brain as a high-performance alternative to a real hand. Lee, etc., [106] demonstrate that it is possible to realise MRI-based realtime control of a robotic arm only through subjects' thought processes.

Although MEG and fMRI are able to provide patterns for controlling external devices, MEG devices and fMRI scanners are expensive, immobile [86], [106]. Moreover, MEG devices are extremely vulnerable to body-generated and urban magnetic noise, when operating outside magnetically shielded rooms [86], [91]. Therefore, MEG and fMRI are not suitable 
for amputees to interact prosthetic hands, except for a great breakthrough of sensing technologies can be achieved.

Conversely, NIRS-based BMIs are inexpensive and portable [107], [108], but no study reports single modal NIRS can capture discriminative hand motion patterns yet. It should be noted that NIR not only can be used as BMIs, but also to identify muscular activities. During muscle contraction, the NIRS feature would show an amplitude change, which is caused by the blood reflux into the muscle [109]. Several researches regarding the fusion of EMG and NIRS for prosthetic control will be discussed in Section VII.

\section{MULTI-SENSORY FUSION}

Multi-sensory fusion provides a feasible strategy for the improvement of HMIs' stability. The inherent properties and characteristics of bio-signals, as shown in Table. II, can be integrated to better interpreting users' intentions. A consistent finding shows that multi-sensory fusion benefits hand motion classification accuracy.

\section{A. Inertial Transducer}

Hand motion estimation by uni-modal EMG signal deteriorates by the factors, like arm postures, weight of the prostheses, etc. As reported by [57], [111], [112], hand motion classification accuracy is strongly dependent on limb position, and a surface recorded EMG pattern caused by the simple lifting or moving of a prosthesis can be misclassified into a hand control movement. Therefore, some studies introduce inertial transducers, like accelerometer, to track the changes of arm positions. Experiments have shown that adding accelerometer signals can improve hand motion classification accuracy [113], [112] rather than adding more EMG channels [27].

In terms of signal fusion, two approaches were utilised in [111]. One is named dual-stage approach, which identifies the hand position using uni-modal accelerometer data, then recognises hand motions on the basis of the hand position information. The other one simply extends the dimension of EMG feature vector by additional accelerometer signal features [27].

In addition, accelerometer signals have also been integrated in MMG based prosthetic control system. In [114], accelerometer signals function to detect muscle contraction by a dynamic threshold on MMG signals.

\section{B. Electromyography and Near Infrared Spectroscopy}

It is well accepted that EMG signals are sensitive to muscle fatigue, which results in faulty classification of hand motions. NIRS is capable of measuring muscle fatigue [115], so it can be utilised to compensate the negative effects of muscle fatigue. Evidence have demonstrated that the combination of EMG and NIR provides better hand motion classification accuracy than uni-modal signals [109], [116], [117]. In the fusion of signals, Herrmann, etc., [109], [116] proposed a feature named NIRSRMS that combines a weighted NIR signal with the RMS value of EMG signals:

$$
N I R S R M S=R M S \times N I R S
$$

\section{Electroencephalograhy and Electroneurography}

Combining non-invasive EEG signal with the invasive recording of the nerve motor output could provide robust natural and bidirectional multi-modal HMIs. Rossini, etc., [118] show that the information gathered from EEG signals is able to significantly improve classification performance based only on ENG signal analysis, which briefly presents the idea that handrelated activities can be decoded by the combined analysis of motor-related signals simultaneously gathered via intraneural electrodes implanted into the PNS and scalp recorded EEG signals to govern a dexterous hand prosthesis. Tombini, etc., [110] also demonstrate that the movement classification performance improves through focusing ENG in an EEG-driven time window.

\section{NEW Challenges AND DiRECTIONS}

\section{A. Robust Sensing Technology}

With the development of sensing technologies, a variety of HMIs can be employed to control robotic prostheses. However, the robustness of bio-signal control still suffers. Some issues can be considered to fulfil robust bio-sensing for prosthetic control. Firstly, electrode displacement is a ubiquitous phenomenon in bio-sensing techniques. The intensity, quality and repeatability of bio-signals depend on correct placement of electrodes. As such, new electrode arrangement approach should be concerned to avoid electrode displacements. Secondly, bio-signals tend to be contaminated by various noises. Thus, indirect approaches can be exploited, like force sensitive resistor (FSR) sensor [31], [119], throat microphone [120], capacitive sensing [121]. Thirdly, remarkable signal changes would happen while subjects carry out finger movements or grasp if they also involve in arm motions. Therefore, advanced sensing techniques should be able to distinguish the target hand motion from interference motions. Fourthly, invasive PNIs, ECoG suffer from signal degradation during a long time use, which promotes further research to improve the long-term reliability of neural-interface systems [122].

\section{B. Multi-modal Sensory Fusion}

Given the difficulty of robust control by uni-modal sensors, multi-modal sensory fusion is a promising substitution. Unimodal sensor suffers from information deficiency and specific noises, and sensory fusion provide a complementary strategy. For instance, EMG signals are susceptible to muscle fatigue while NIR can diagnose it, and thus NIR sensor can be integrated into EMG systems for fatigue to compensate the shortage of EMG. Some works of multi-modal sensory fusion have been introduced in Section VII. However, these works just simply employ two or several types of sensory systems to collect data for off-line analysis. Further direction of multimodal sensory fusion is to integrate them in both hardware and software. Miniature sensors and embedded systems with considerable processing power are currently available so that the integration is feasible [10]. 
TABLE II. CATEGORISATION AND COMPARISON OF HUMAN-MACHINE INTERFACES

\begin{tabular}{cccc}
\hline Categrates & Bio-signals & Bio-information & Physical Properties \\
\hline \multirow{3}{*}{ Muscle-machine interface } & EMG [10] & Muscle action potential & Electric \\
& SMG [64] & Muscle morphology & Ultrasonic \\
& MMG [73] & Muscle oscillation & Acoustic \\
& NIRS [109] & Oxygenation & Infrared \\
\hline \multirow{2}{*}{ Neuro-machine interface } & ENG [78], [77] & Peripheral neuronal activation & Electric \\
\hline \multirow{2}{*}{ Brain-machine interface } & EEG [110] & Brain neuronal activation & Electric \\
& ECoG [77] & Brain neuronal activation & Electric \\
\hline
\end{tabular}

\section{On-line Signal Processing and Learning Algorithms}

Most of studies towards bio-signal based hand motion analysis and prosthetic control algorithms are implemented in a controlled laboratory environment, and subjects sit with a comfortable arm position without moving limbs. Therefore, the bio-signals cannot reflect the real situation when amputees using a prosthesis in daily life. In addition, a motion pattern that obtained in the training session would severely mismatch the real patterns of the same intended motion. Future systems should be able to deal with bio-signals under varying conditions, such as free-to-move residual limb, diverse physical factors, artefacts interference, etc. With such uncertainties, offline analysis would be little impact on the improvement of HMIs for prosthetic control, because the limited off-line data are not able to manifest the dynamic characteristics of biosignals in practice. Adaptive signal processing of the sensory data stream is an important aspect for on-line systems, as discussed in [10].

\section{Bio-feedback}

Decoding bio-signals into control commands of prostheses is a feedforward path. Vision is the natural feedback path that is utilised during robotic hand manipulation by amputees. However, the human hand not only has proprioception, but also owns tactile, pressure, stiffness and temperature sensations. These multi-modal feelings are comprehensively incepted by the "human body controller" to adjust hand gestures and grasps. Therefore, it is necessary to bring in multi-modal sensory feedbacks to robotic hands and make the prosthesis a sensible replacement of a lost hand [2].

On the one hand, advanced sensors should be developed to mimic the sensing functions of the human hand such as high resolution pressure/haptic sensing array; on the other hand, novel approaches are required to integrate the feedback information into the perception system of amputees to enhance prosthesis ownership. Targeted sensory reinnervation [123], TMR [124], intracortical microstimulation [125] and nerve stimulation [84], [85] have been proved feasible to activate the perception system of humans. To avoid surgery of the above methods, feedback information can be transferred to the less functional skin by vibrotactile [126], mechanotactile [127] and electrotactile [128] stimulation. Further directions can be focused on: direct neural interfaces that provide intuitive sensory feedback; interactive HMIs enables humans to control hardware and collect feedback information [129]; techniques that separate the feedback signals with the feedforward signals
[10]; training strategies that allow amputees to fit in the artificial control system with bio-feedback [130].

\section{CONCLUSION}

This paper investigates a variety of HMIs for interacting hand prostheses. The sensing techniques, including EMG, SMG, MMG, EEG, ECoG, ENG, etc., are introduced in the big background of hand motion classification, finger/wrist angle prediction and prosthetic hand manipulation. EMG that measures the summation of individual action potentials is mostly employed in the control of hand prostheses, where the key issues of electrode configuration, PR based prosthetic control, on-line training are discussed. BMIs and PNIs have also been explored to analyse the relationship between human hand motions and brain or nervous activities. However, the long term usability and robustness of BMIs and PNIs should be improved before they can be applied in practice. In addition, SMG monitoring morphological changes in forearm muscles and MMG measuring lateral oscillations generated by dimensional changes in active muscle fibres are two physical approaches, which are immune to electromagnetic interferences. Moreover, this paper also involves the latest multimodal sensing techniques. It is verified that the combinations of EMG and accelerometers, EMG and NIRS, EEG and ENG can yield better hand gesture recognition accuracy than unimodal signals. In the end, several challenges and directions are concluded.

\section{REFERENCES}

[1] G. C. Matrone, C. Cipriani, E. L. Secco, G. Magenes, and M. C. Carrozza, "Principal components analysis based control of a multi-dof underactuated prosthetic hand," J. Neuroeng. Rehabil., vol. 7, p. 16, 2010.

[2] C. Castellini and P. V. D. Smagt, "Surface emg in advanced hand prosthetics," Biol. Cybern., vol. 100, no. 1, pp. 35-47, 2009.

[3] C. L. Pulliam, J. M. Lambrecht, and R. F. Kirsch, "Electromyogrambased neural network control of transhumeral prostheses," J. Rehabil. Res. Dev., vol. 48, no. 6, pp. 739-54, 2011.

[4] C. Lake and R. Dodson, "Progressive upper limb prosthetics," Phys. Med. Rehabil. Clin. N. Am., vol. 17, no. 1, pp. 49-72, 2006.

[5] F. V. G. Tenore, A. Ramos, A. Fahmy, S. Acharya, R. EtienneCummings, and N. V. Thakor, "Decoding of individuated finger movements using surface electromyography," IEEE T. Biomed. Eng, vol. 56, no. 5, pp. 1427-1434, 2009.

[6] S. P. Arjunan and D. K. Kumar, "Decoding subtle forearm flexions using fractal features of surface electromyogram from single and multiple sensors," J. Neuroeng. Rehabil., vol. 7, p. 53, 2010. 
[7] C. Cipriani, C. Antfolk, M. Controzzi, G. Lundborg, B. Rosen, M. C. Carrozza, and F. Sebelius, "Online myoelectric control of a dexterous hand prosthesis by transradial amputees," IEEE T. Neural Syst. Rehabil. Eng., vol. 19, no. 3, pp. 260-270, 2011.

[8] F. C. Sebelius, B. N. Rosen, and G. N. Lundborg, "Refined myoelectric control in below-elbow amputees using artificial neural networks and a data glove," J. Hand Surg. Am., vol. 30, no. 4, pp. 780-9, 2005.

[9] M. Khezri and M. Jahed, "An exploratory study to design a novel hand movement identification system," Comput. Biol. Med., vol. 39, no. 5, pp. 433-42, 2009.

[10] N. Jiang, S. Dosen, K. R. Muller, and D. Farina, "Myoelectric control of artificial limbs-is there a need to change focus?" IEEE Signal Process. Mag., vol. 29, no. 5, pp. 147-150, 2012.

[11] C. Cipriani, J. L. Segil, J. A. Birdwell, and R. F. F. Weir, "Dexterous control of a prosthetic hand using fine-wire intramuscular electrodes in targeted extrinsic muscles," IEEE T. Syst. Rehabil. Eng., vol. 22, no. 4, pp. 828-836, 2014

[12] E. N. Kamavuako, J. C. Rosenvang, M. F. Bg, A. Smidstrup, E. Erkocevic, M. J. Niemeier, W. Jensen, and D. Farina, "Influence of the feature space on the estimation of hand grasping force from intramuscular emg," Biomed. Signal Proces., vol. 8, no. 1, pp. 1-5, 2013.

[13] L. J. Hargrove, K. Englehart, and B. Hudgins, "A comparison of surface and intramuscular myoelectric signal classification," IEEE T. Biomed. Eng, vol. 54, no. 5, pp. 847-853, 2007.

[14] H. S. Ryait, A. S. Arora, and R. Agarwal, "Study of issues in the development of surface emg controlled human hand," J. Mater. Sci. Mater. Med., vol. 20 Suppl 1, pp. S107-14, 2009.

[15] J. Rafiee, M. A. Rafiee, F. Yavari, and M. P. Schoen, "Feature extraction of forearm emg signals for prosthetics," Expert. Syst. Appl., vol. 38, no. 4, pp. 4058-4067, 2011.

[16] A. Phinyomark, F. Quaine, S. Charbonnier, C. Serviere, F. TarpinBernard, and Y. Laurillau, "Emg feature evaluation for improving myoelectric pattern recognition robustness," Expert. Syst. Appl., vol. 40 no. 12 , pp. $4832-4840,2013$

[17] A. Andrews, E. Morin, and L. McLean, "Optimal electrode configurations for finger movement classification using emg," Conf. Proc. IEEE Eng. Med. Biol. Soc., vol. 2009, pp. 2987-90, 2009.

[18] G. Li, Y. Geng, D. Tao, and P. Zhou, "Performance of electromyography recorded using textile electrodes in classifying arm movements," Conf. Proc. IEEE Eng. Med. Biol. Soc., vol. 2011, pp. 4243-6, 2011.

[19] Y. H. Chiou, J. J. Luh, S. C. Chen, J. S. Lai, and T. S. Kuo, "The comparison of electromyographic pattern classifications with active and passive electrodes," Med. Eng. Phys., vol. 26, no. 7, pp. 605-10, 2004.

[20] U. Baspinar, H. S. Varol, and V. Y. Senyurek, "Performance comparison of artificial neural network and gaussian mixture model in classifying hand motions by using semg signals," Biocybern. Biomed. Eng., vol. 33, no. 1, pp. 33-45, 2013.

[21] N. M. Kakoty and S. M. Hazarika, "Recognition of grasp types through principal components of dwt based emg features," IEEE Int. Conf. Rehabil. Robot, vol. 2011, p. 5975398, 2011.

[22] C. Castellini, E. Gruppioni, A. Davalli, and G. Sandini, "Fine detection of grasp force and posture by amputees via surface electromyography," J. Physiol. Paris., vol. 103, no. 3-5, pp. 255-62, 2009.

[23] G. Huang, Z. Zhang, D. Zhang, and X. Zhu, "Spatio-spectral filters for low-density surface electromyographic signal classification," Med. Biol. Eng. Comput., vol. 51, no. 5, pp. 547-55, 2013.

[24] G. Drost, D. F. Stegeman, B. G. van Engelen, and M. J. Zwarts, "Clinical applications of high-density surface emg: a systematic review," J. Electromyogr. Kines., vol. 16, no. 6, pp. 586-602, 2006.

[25] S. Muceli and D. Farina, "Simultaneous and proportional estimation of hand kinematics from emg during mirrored movements at multiple degrees-of-freedom,” IEEE T. Syst. Rehabil. Eng., vol. 20, no. 3, pp. 371-8, 2012.
[26] H. Daley, K. Englehart, L. Hargrove, and U. Kuruganti, "High density electromyography data of normally limbed and transradial amputee subjects for multifunction prosthetic control,' J. Electromyogr. Kines., vol. 22, no. 3, pp. 478-84, 2012.

[27] A. Fougner, E. Scheme, A. D. Chan, K. Englehart, and O. Stavdahl, "A multi-modal approach for hand motion classification using surface emg and accelerometers," Conf. Proc. IEEE Eng. Med. Biol. Soc., vol. 2011, pp. 4247-50, 2011.

[28] R. N. Khushaba, A. Al-Ani, and A. Al-Jumaily, "Orthogonal fuzzy neighborhood discriminant analysis for multifunction myoelectric hand control," IEEE T. Biomed. Eng, vol. 57, no. 6, pp. 1410-9, 2010.

[29] F. Tenore, A. Ramos, A. Fahmy, S. Acharya, R. Etienne-Cummings, and N. V. Thakor, "Towards the control of individual fingers of a prosthetic hand using surface emg signals," Conf. Proc. IEEE Eng. Med. Biol. Soc., vol. 2007, pp. 6146-9, 2007.

[30] A. J. Young, L. J. Hargrove, and T. A. Kuiken, "Improving myoelectric pattern recognition robustness to electrode shift by changing interelectrode distance and electrode configuration," IEEE T. Biomed. Eng, vol. 59, no. 3, pp. 645-652, 2012.

[31] N. Li, D. Yang, L. Jiang, H. Liu, and H. Cai, "Combined use of fsr sensor array and svm classifier for finger motion recognition based on pressure distribution map," J. Bionic. Eng., vol. 9, no. 1, pp. 39-47, 2012.

[32] H. He, Z. Ping, L. Guanglin, and T. A. Kuiken, "An analysis of emg electrode configuration for targeted muscle reinnervation based neural machine interface," IEEE T. Syst. Rehabil. Eng., vol. 16, no. 1, pp. 37-45, 2008

[33] M. Atzori, A. Gijsberts, S. Heynen, A. G. Hager, C. Castellimi, B. Caputo, and H. Muller, "Experiences in the creation of an electromyography database to help hand amputated persons," Stud. Health. Technol. Inform., vol. 180, pp. 828-32, 2012.

[34] P. Zhou, M. M. Lowery, K. B. Englehart, H. Huang, G. Li, L. Hargrove, J. P. Dewald, and T. A. Kuiken, "Decoding a new neural machine interface for control of artificial limbs," J. Neurophysiol., vol. 98, no. 5, pp. 2974-82, 2007.

[35] A. Fougner, O. Stavdahl, P. J. Kyberd, Y. G. Losier, and P. A. Parker, "Control of upper limb prostheses: Terminology and proportional myoelectric control; a review," IEEE T. Syst. Rehabil. Eng., vol. 20, no. 5, pp. 663-677, 2012.

[36] D. Farina, N. Jiang, H. Rehbaum, A. Holobar, B. Graimann, H. Dietl, and O. C. Aszmann, "The extraction of neural information from the surface emg for the control of upper-limb prostheses: Emerging avenues and challenges," IEEE T. Syst. Rehabil. Eng., vol. 22, no. 4, pp. 797-809, 2014

[37] M. A. Oskoei and H. S. Hu, "Myoelectric control systems-a survey," Biomed. Signal Proces., vol. 2, no. 4, pp. 275-294, 2007.

[38] S. Amsuss, P. M. Goebel, J. Ning, B. Graimann, L. Paredes, and D. Farina, "Self-correcting pattern recognition system of surface emg signals for upper limb prosthesis control," IEEE T. Biomed. Eng, vol. 61, no. 4, pp. 1167-1176, 2014.

[39] E. J. Scheme, K. B. Englehart, and B. S. Hudgins, "Selective classification for improved robustness of myoelectric control under nonideal conditions," IEEE T. Biomed. Eng, vol. 58, no. 6, pp. 1698-1705, 2011

[40] X. Chen, D. Zhang, and X. Zhu, "Application of a self-enhancing classification method to electromyography pattern recognition for multifunctional prosthesis control," J. Neuroeng. Rehabil., vol. 10, no. 1, p. 44, 2013.

[41] S. A. Dalley, D. A. Bennett, and M. Goldfarb, "Preliminary functional assessment of a multigrasp myoelectric prosthesis," Conf. Proc. IEEE Eng. Med. Biol. Soc., vol. 2012, pp. 4172-5, 2012.

[42] M. Khezri and M. Jahed, "Real-time intelligent pattern recognition algorithm for surface emg signals," Biomed. Eng. Online, vol. 6, p. 45, 2007.

[43] K. Momen, S. Krishnan, and T. Chau, "Real-time classification of 
forearm electromyographic signals corresponding to user-selected intentional movements for multifunction prosthesis control," IEEE T. Neural Syst. Rehabil. Eng., vol. 15, no. 4, pp. 535-542, 2007.

[44] J. Zhong, J. Shi, Y. Cai, and Q. Zhang, "Recognition of hand motions via surface emg signal with rough entropy," Conf. Proc. IEEE Eng. Med. Biol. Soc., vol. 2011, pp. 4100-3, 2011.

[45] E. C. Orosco, N. M. Lopez, and F. di Sciascio, "Bispectrum-based features classification for myoelectric control," Biomed. Signal Proces., vol. 8, no. 2, pp. 153-168, 2013.

[46] P. Geethanjali and K. K. Ray, "Identification of motion from multichannel emg signals for control of prosthetic hand," Australas Phys. Eng. Sci. Med., vol. 34, no. 3, pp. 419-27, 2011.

[47] M. A. Powell, R. R. Kaliki, and N. V. Thakor, "User training for pattern recognition-based myoelectric prostheses: Improving phantom limb movement consistency and distinguishability," IEEE T. Syst. Rehabil. Eng., vol. 22, no. 3, pp. 522-532, 2014.

[48] D. Yang, J. Zhao, Y. Gu, X. Wang, N. Li, L. Jiang, H. Liu, H. Huang, and D. Zhao, "An anthropomorphic robot hand developed based on underactuated mechanism and controlled by emg signals," J. Bionic. Eng., vol. 6, no. 3, pp. 255-263, 2009.

[49] Y. C. Du, C. H. Lin, L. Y. Shyu, and T. S. Chen, "Portable hand motion classifier for multi-channel surface electromyography recognition using grey relational analysis," Expert. Syst. Appl., vol. 37, no. 6, pp. 4283-4291, 2010.

[50] R. N. Khushaba, A. Al-Jumaily, and A. Al-Ani, "Evolutionary fuzzy discriminant analysis feature projection technique in myoelectric control," Pattern Recogn. Lett., vol. 30, no. 7, pp. 699-707, 2009.

[51] _ " "Fuzzy discriminant analysis based feature projection in myoelectric control," Conf. Proc. IEEE Eng. Med. Biol. Soc., vol. 2008, pp. 5049-52, 2008 .

[52]

[53] P. M. Pilarski, M. R. Dawson, T. Degris, J. P. Carey, K. M. Chan, J. S. Hebert, and R. S. Sutton, "Adaptive artificial limbs a real-time approach to prediction and anticipation," IEEE Robot Autom. Mag., vol. 20, no. 1, pp. 53-64, 2013.

[54] L. A. Miller, R. D. Lipschutz, K. A. Stubblefield, B. A. Lock, H. Huang, r. Williams, T. W., R. F. Weir, and T. A. Kuiken, "Control of a six degree of freedom prosthetic arm after targeted muscle reinnervation surgery," Arch Phys Med Rehabil, vol. 89, no. 11, pp. 2057-65, 2008.

[55] J. M. Hahne, F. Biebmann, N. Jiang, H. Rehbaum, D. Farina, F. C. Meinecke, K. R. Muller, and L. C. Parra, "Linear and nonlinear regression techniques for simultaneous and proportional myoelectric control," IEEE T. Syst. Rehabil. Eng., vol. 22, no. 2, pp. 269-279, 2014.

[56] A. B. Ajiboye and R. F. Weir, "Muscle synergies as a predictive framework for the emg patterns of new hand postures," J. Neural Eng., vol. 6, no. 3, p. 036004, 2009.

[57] C. Cipriani, R. Sassu, M. Controzzi, and M. C. Carrozza, "Influence of the weight actions of the hand prosthesis on the performance of pattern recognition based myoelectric control: preliminary study," Conf. Proc. IEEE Eng. Med. Biol. Soc., vol. 2011, pp. 1620-3, 2011.

[58] H. Bouwsema, C. K. Van, and R. M. Bongers, "Learning to control opening and closing a myoelectric hand," Archives of Physical Medicine and Rehabilitation, vol. 91, no. 9, pp. 1442-1446, 2010.

[59] T. A. Kuiken, G. A. Dumanian, R. D. Lipschutz, L. A. Miller, and K. A. Stubblefield, "The use of targeted muscle reinnervation for improved myoelectric prosthesis control in a bilateral shoulder disarticulation amputee," Prosthet. Orthot. Int., vol. 28, no. 3, pp. 24553, 2004.

[60] T. A. Kuiken, L. A. Miller, R. D. Lipschutz, B. A. Lock, K. Stubblefield, P. D. Marasco, P. Zhou, and G. A. Dumanian, "Targeted reinnervation for enhanced prosthetic arm function in a woman with a proximal amputation: a case study," Lancet, vol. 369, no. 9559, pp. 371-80, 2007.
[61] C. Castellini, G. Passig, and E. Zarka, "Using ultrasound images of the forearm to predict finger positions," IEEE T. Syst. Rehabil. Eng., vol. 20, no. 6, pp. 788-797, 2012.

[62] P. L. Cooperberg, I. Tsang, L. Truelove, and W. J. Knickerbocker, "Gray scale ultrasound in the evaluation of rheumatoid arthritis of the knee," Radiology, vol. 126, no. 3, pp. 759-63, 1978.

[63] K. Fukumoto, S. Muraki, M. Tsubai, and O. Fukuda, "Calibration of cross-sectional images measured by an ultrasound-based muscle evaluation system," Conf. Proc. IEEE Eng. Med. Biol. Soc., pp. 428431, 2009.

[64] Y.-P. Zheng, M. Chan, J. Shi, X. Chen, and Q.-H. Huang, "Sonomyography: Monitoring morphological changes of forearm muscles in actions with the feasibility for the control of powered prosthesis," Med. Eng. Phys., vol. 28, no. 5, pp. 405-415, 2006.

[65] Q. H. Huang, Y. P. Zheng, X. Chena, J. F. He, and J. Shi, "A system for the synchronized recording of sonomyography, electromyography and joint angle," Open Biomed. Eng. J., vol. 1, pp. 77-84, 2007.

[66] E. R. Zarka, "Prediction of finger movements using ultrasound images," Thesis, 2011.

[67] J. Shi, S. Hu, Z. Liu, J. Guo, Y. Zhou, and Y. Zheng, "Recognition of finger flexion from ultrasound image with optical flow: A preliminary study," Int. Conf. Biomedical Engineering and Computer Science, pp. $1-4,2010$.

[68] S. Sikdar, H. Rangwala, E. B. Eastlake, I. A. Hunt, A. J. Nelson, J. Devanathan, A. Shin, and J. J. Pancrazio, "Novel method for predicting dexterous individual finger movements by imaging muscle activity using a wearable ultrasonic system," IEEE T. Neural Syst. Rehabil. Eng., vol. 22, no. 1, pp. 69-76, 2014.

[69] Q. Chang, J. Shi, Z. Xiao, and Y. Zheng, "A research of smg controlled prosthetic hand with sse2 acceleration," Int. Conf. Signal Processing, pp. 2146-2149, 2008.

[70] Y. Tsutsui, Y. Sakata, T. Tanaka, S. Kaneko, and M. Q. Feng, "Human joint movement recognition by using ultrasound echo based on test feature classifier," 2007 IEEE Sensors, pp. 1205-1208, 2007.

[71] D. Ni, X. Chen, W. Yi, Y. Zheng, Z. Zhu, and S. Chan, "In vivo behavior of human muscle during isometric ramp contraction: A simultaneous emg, mmg and ultrasonography investigation," IEEE Int. Conf. Signal Processing, Communication and Computing, pp. 59-62, 2012.

[72] J. P. Cole, G. Madhavan, and K. J. McLeod, "Vibromyographic quantification of voluntary isometric contractile force in the brachioradialis," Conf. Proc. IEEE Eng. Med. Biol. Soc., pp. 1708-1710, 2006.

[73] N. Alves and T. Chau, "Vision-based segmentation of continuous mechanomyographic grasping sequences," IEEE T. Biomed. Eng, vol. 55, no. 2, pp. 765-773, 2008.

[74] K. Lei, W. Tsai, W. Lin, and M. Lee, "Mmg-torque estimation under dynamic contractions," IEEE int. conf. Systems, Man, and Cybernetics, pp. 585-590, 2011.

[75] J. Silva, W. Heim, and T. Chau, "Mmg-based classification of muscle activity for prosthesis control," Conf. Proc. IEEE Eng. Med. Biol. Soc., vol. 1, pp. 968-971, 2004.

[76] Y. Zeng, Z. Yang, W. Cao, and C. Xia, "Hand-motion patterns recognition based on mechanomyographic signal analysis," Int. Conf. Future BioMedical Information Engineering, pp. 21-24, 2009.

[77] S. Micera, P. M. Rossini, J. Rigosa, L. Citi, J. Carpaneto, S. Raspopovic, M. Tombini, C. Cipriani, G. Assenza, M. C. Carrozza, K. P. Hoffmann, K. Yoshida, X. Navarro, and P. Dario, "Decoding of grasping information from neural signals recorded using peripheral intrafascicular interfaces," J. Neuroeng. Rehabil., vol. 8, p. 53, 2011.

[78] J. Carpaneto, A. Cutrone, S. Bossi, P. Sergi, L. Citi, J. Rigosa, P. M. Rossini, and S. Micera, "Activities on pns neural interfaces for the control of hand prostheses," Conf. Proc. IEEE Eng. Med. Biol. Soc., vol. 2011, pp. 4637-40, 2011.

[79] K. Ohnishi, R. F. Weir, and T. A. Kuiken, "Neural machine interfaces 
for controlling multifunctional powered upper-limb prostheses," Expert Rev Med Devices, vol. 4, no. 1, pp. 43-53, 2007.

[80] X. Navarro, T. B. Krueger, N. Lago, S. Micera, T. Stieglitz, and P. Dario, "A critical review of interfaces with the peripheral nervous system for the control of neuroprostheses and hybrid bionic systems," J. Peripher. Nerv. Syst., vol. 10, no. 3, pp. 229-258, 2005.

[81] S. Micera, J. Rigosa, J. Carpaneto, L. Citi, S. Raspopovic, E. Guglielmelli, A. Benvenuto, L. Rossini, G. Di Pino, G. Cavallo, M. C. Carrozza, C. Cipriani, K. P. Hoffmann, P. Dario, and P. M. Rossini, "On the control of a robot hand by extracting neural signals from the pns: preliminary results from a human implantation," Conf. Proc. IEEE Eng. Med. Biol. Soc., vol. 2009, pp. 4586-9, 2009.

[82] N. V. Thakor and J. He, "Guest editorial special theme on neural and myoelectric control of prostheses," IEEE T. Syst. Rehabil. Eng., vol. 16 , no. 1 , pp. $1-2,2008$.

[83] G. S. Dhillon and K. W. Horch, "Direct neural sensory feedback and control of a prosthetic arm," IEEE T. Syst. Rehabil. Eng., vol. 13, no. 4, pp. 468-72, 2005.

[84] G. S. Dhillon, T. B. Kruger, J. S. Sandhu, and K. W. Horch, "Effects of short-term training on sensory and motor function in severed nerves of long-term human amputees," J. Neurophysiol., vol. 93, no. 5, pp. 2625-2633, 2005.

[85] P. M. Rossini, S. Micera, A. Benvenuto, J. Carpaneto, G. Cavallo, L. Citi, C. Cipriani, L. Denaro, V. Denaro, G. Di Pino, F. Ferreri, E. Guglielmelli, K. P. Hoffmann, S. Raspopovic, J. Rigosa, L. Rossini, M. Tombini, and P. Dario, "Double nerve intraneural interface implant on a human amputee for robotic hand control," Clin. Neurophysiol., vol. 121, no. 5, pp. 777-83, 2010.

[86] O. Tonet, M. Marinelli, L. Citi, P. M. Rossini, L. Rossini, G. Megali, and P. Dario, "Defining brain-machine interface applications by matching interface performance with device requirements," J. Neurosci. Methods, vol. 167, no. 1, pp. 91-104, 2008.

[87] J. L. Collinger, B. Wodlinger, J. E. Downey, W. Wang, E. C. TylerKabara, D. J. Weber, A. J. C. McMorland, M. Velliste, M. L. Boninger, and A. B. Schwartz, "High-performance neuroprosthetic control by an individual with tetraplegia," The Lancet, vol. 381, no. 9866, pp. 557564, 2012.

[88] Y. Gu, K. Dremstrup, and D. Farina, "Single-trial discrimination of type and speed of wrist movements from eeg recordings," Clin. Neurophysiol., vol. 120, no. 8, pp. 1596-600, 2009.

[89] G. Buzski, C. A. Anastassiou, and C. Koch, "The origin of extracellular fields and currentseeg, ecog, lfp and spikes," Nat. Rev. Neurosci., vol. 13, no. 6, pp. 407-420, 2012.

[90] J. Yang, H. Singh, E. L. Hines, F. Schlaghecken, D. D. Iliescu, M. S. Leeson, and N. G. Stocks, "Channel selection and classification of electroencephalogram signals: An artificial neural network and genetic algorithm-based approach," Artif. Intell. Med., vol. 55, no. 2, pp. $117-$ 126, 2012.

[91] T. Yanagisawa, M. Hirata, Y. Saitoh, T. Goto, H. Kishima, R. Fukuma, H. Yokoi, Y. Kamitani, and T. Yoshimine, "Real-time control of a prosthetic hand using human electrocorticography signals," J. Neurosurg., vol. 114, no. 6, pp. 1715-1722, 2011.

[92] L. Kauhanen, T. Nykopp, J. Lehtonen, P. Jylanki, J. Heikkonen, P. Rantanen, H. Alaranta, and M. Sams, "Eeg and meg brain-computer interface for tetraplegic patients," IEEE T. Syst. Rehabil. Eng., vol. 14, no. 2, pp. 190-3, 2006.

[93] H. A. Agashe and J. L. Contreras-Vidal, "Reconstructing hand kinematics during reach to grasp movements from electroencephalographic signals," Conf. Proc. IEEE Eng. Med. Biol. Soc., vol. 2011, pp. 5444 7, 2011.

[94] A. K. Mohamed, T. Marwala, and L. R. John, "Single-trial eeg discrimination between wrist and finger movement imagery and execution in a sensorimotor bci," Conf. Proc. IEEE Eng. Med. Biol. Soc., vol. 2011, pp. 6289-93, 2011.

[95] T. J. Bradberry, R. J. Gentili, and J. L. Contreras-Vidal, "Decoding three-dimensional hand kinematics from electroencephalographic sig- nals," Conf. Proc. IEEE Eng. Med. Biol. Soc., vol. 2009, pp. 5010-3, 2009.

[96] M. S. Fifer, S. Acharya, H. L. Benz, M. Mollazadeh, N. E. Crone, and N. V. Thakor, "Toward electrocorticographic control of a dexterous upper limb prosthesis: building brain-machine interfaces," IEEE Pulse, vol. 3, no. 1, pp. 38-42, 2012.

[97] T. Yanagisawa, M. Hirata, Y. Saitoh, H. Kishima, K. Matsushita, T. Goto, R. Fukuma, H. Yokoi, Y. Kamitani, and T. Yoshimine, "Electrocorticographic control of a prosthetic arm in paralyzed patients," Ann. Neurol., vol. 71, no. 3, pp. 353-61, 2012.

[98] C. A. Chestek, V. Gilja, C. H. Blabe, B. L. Foster, K. V. Shenoy, J. Parvizi, and J. M. Henderson, "Hand posture classification using electrocorticography signals in the gamma band over human sensorimotor brain areas," J. Neural Eng., vol. 10, no. 2, p. 026002, 2013.

[99] H. L. Benz, Z. Huaijian, A. Bezerianos, S. Acharya, N. E. Crone, Z. Xioaxiang, and N. V. Thakor, "Connectivity analysis as a novel approach to motor decoding for prosthesis control," IEEE T. Syst. Rehabil. Eng., vol. 20, no. 2, pp. 143-152, 2012.

[100] L. R. Hochberg, M. D. Serruya, G. M. Friehs, J. A. Mukand, M. Saleh, A. H. Caplan, A. Branner, D. Chen, R. D. Penn, and J. P. Donoghue, "Neuronal ensemble control of prosthetic devices by a human with tetraplegia," Nature, vol. 442, no. 7099, pp. 164-171, 2006.

[101] L. R. Hochberg, D. Bacher, B. Jarosiewicz, N. Y. Masse, J. D. Simeral, J. Vogel, S. Haddadin, J. Liu, S. S. Cash, and P. van der Smagt, "Reach and grasp by people with tetraplegia using a neurally controlled robotic arm," Nature, vol. 485, no. 7398, pp. 372-375, 2012.

[102] H. Sugata, T. Goto, M. Hirata, T. Yanagisawa, M. Shayne, K. Matsushita, T. Yoshimine, and S. Yorifuji, "Neural decoding of unilateral upper limb movements using single trial meg signals," Brain Res., vol. 1468, pp. 29-37, 2012.

[103] L. Kauhanen, T. Nykopp, and M. Sams, "Classification of single meg trials related to left and right index finger movements," Clin. Neurophysiol., vol. 117, no. 2, pp. 430-9, 2006.

[104] F. Quandt, C. Reichert, H. Hinrichs, H. J. Heinze, R. T. Knight, and J. W. Rieger, "Single trial discrimination of individual finger movements on one hand: a combined meg and eeg study," NeuroImage, vol. 59, no. 4, pp. 3316-24, 2012.

[105] M. Maruishi, Y. Tanaka, H. Muranaka, T. Tsuji, Y. Ozawa, S. Imaizumi, M. Miyatani, and J. Kawahara, "Brain activation during manipulation of the myoelectric prosthetic hand: a functional magnetic resonance imaging study," NeuroImage, vol. 21, no. 4, pp. 1604-11, 2004.

[106] J. Lee, J. Ryu, F. A. Jolesz, Z. Cho, and S. Yoo, "Brainmachine interface via real-time fmri: Preliminary study on thought-controlled robotic arm," Neurosci. Lett., vol. 450, no. 1, pp. 1-6, 2009.

[107] S. Coyle, T. Ward, C. Markham, and G. McDarby, "On the suitability of near-infrared (nir) systems for next-generation brain-computer interfaces," Physiol. Meas., vol. 25, no. 4, pp. 815-22, 2004.

[108] S. K. Piper, A. Krueger, S. P. Koch, J. Mehnert, C. Habermehl, J. Steinbrink, H. Obrig, and C. H. Schmitz, "A wearable multi-channel fnirs system for brain imaging in freely moving subjects," NeuroImage, vol. 85, pp. 64-71, 2014.

[109] S. Herrmann, A. Attenberger, and K. Buchenrieder, "Prostheses control with combined near-infrared and myoelectric signals," Computer Aided Systems TheoryEUROCAST 2011, pp. 601-608, 2012.

[110] M. Tombini, J. Rigosa, F. Zappasodi, C. Porcaro, L. Citi, J. Carpaneto, P. M. Rossini, and S. Micera, "Combined analysis of cortical (eeg) and nerve stump signals improves robotic hand control," Neurorehabil. Neural Repair, vol. 26, no. 3, pp. 275-81, 2012.

[111] E. Scheme, A. Fougner, O. Stavdahl, A. C. Chan, and K. Englehart, "Examining the adverse effects of limb position on pattern recognition based myoelectric control," Conf. Proc. IEEE Eng. Med. Biol. Soc., vol. 2010, pp. 6337-40, 2010.

[112] A. Fougner, E. Scheme, A. D. C. Chan, K. Englehart, and O. Stavdahl, 
"Resolving the limb position effect in myoelectric pattern recognition," IEEE T. Syst. Rehabil. Eng., vol. 19, no. 6, pp. 644-651, 2011.

[113] A. Gijsberts, M. Atzori, C. Castellini, H. Muller, and B. Caputo, "Movement error rate for evaluation of machine learning methods for semg-based hand movement classification," IEEE T. Syst. Rehabil. Eng., vol. 22, no. 4, pp. 735-744, 2014.

[114] J. Silva, T. Chau, and A. Goldenberg, "Mmg-based multisensor data fusion for prosthesis control," Conf. Proc. IEEE Eng. Med. Biol. Soc., vol. 3, pp. 2909-2912, 2003.

[115] A. Sakudo, H. Kuratsune, T. Kobayashi, S. Tajima, Y. Watanabe, and K. Ikuta, "Spectroscopic diagnosis of chronic fatigue syndrome by visible and near-infrared spectroscopy in serum samples," Biochem. Bioph. Res. Co., vol. 345, no. 4, pp. 1513-1516, 2006.

[116] S. Herrmann and K. Buchenrieder, "Fusion of myoelectric and near-infrared signals for prostheses control," Proc. Int. Cov. on Rehabilitation Engineering and Assistive Technology, p. 54, 2010.

[117] A. Attenberger and K. Buchenrieder, "Modeling and visualization of classification-based control schemes for upper limb prostheses," Int. Conference and Workshops on Engineering of Computer Based Systems, pp. 188-194, 2012.

[118] L. Rossini and P. M. Rossini, "Combining eng and eeg integrated analysis for better sensitivity and specificity of neuroprosthesis operations," Conf. Proc. IEEE Eng. Med. Biol. Soc., vol. 2010, pp. 134-7, 2010.

[119] Y. Honda, S. Weber, and T. C. Lueth, "Stability analysis for tactile pattern based recognition system for hand gestures," Conf. Proc. IEEE Eng. Med. Biol. Soc., vol. 2007, pp. 4033-6, 2007.

[120] E. Mainardi and A. Davalli, "Controlling a prosthetic arm with a throat microphone," Conf. Proc. IEEE Eng. Med. Biol. Soc., vol. 2007, pp. 3035-9, 2007.

[121] E. Zheng, L. Wang, K. Wei, and Q. Wang, "A non-contact capacitive sensing system for recognizing locomotion modes of transtibial amputees," IEEE T. Biomed. Eng, 2014.

[122] J. W. Judy, "Neural interfaces for upper-limb prosthesis control: opportunities to improve long-term reliability," IEEE Pulse, vol. 3, no. 2, pp. 57-60, 2012.

[123] J. S. Hebert, J. L. Olson, M. J. Morhart, M. R. Dawson, P. D. Marasco, T. A. Kuiken, and K. M. Chan, "Novel targeted sensory reinnervation technique to restore functional hand sensation after transhumeral amputation," IEEE T. Syst. Rehabil. Eng., vol. 22, no. 4, pp. 765773, 2014.

[124] K. Keehoon and J. E. Colgate, "Haptic feedback enhances grip force control of semg-controlled prosthetic hands in targeted reinnervation amputees," IEEE T. Syst. Rehabil. Eng., vol. 20, no. 6, pp. 798-805, 2012.

[125] J. A. Berg, J. F. Dammann, F. V. Tenore, G. A. Tabot, J. L. Boback, L. R. Manfredi, M. L. Peterson, K. D. Katyal, M. S. Johannes, A. Makhlin, R. Wilcox, R. K. Franklin, R. J. Vogelstein, N. G. Hatsopoulos, and S. J. Bensmaia, "Behavioral demonstration of a somatosensory neuroprosthesis," IEEE T. Syst. Rehabil. Eng., vol. 21, no. 3, pp. 500-507, 2013.

[126] M. D’Alonzo, F. Clemente, and C. Cipriani, "Vibrotactile stimulation promotes embodiment of an alien hand in amputees with phantom sensations," IEEE T. Syst. Rehabil. Eng., vol. PP, no. 99, pp. 1-1, 2014.

[127] C. Antfolk, M. D’Alonzo, M. Controzzi, G. Lundborg, B. Rosen, F. Sebelius, and C. Cipriani, "Artificial redirection of sensation from prosthetic fingers to the phantom hand map on transradial amputees: Vibrotactile versus mechanotactile sensory feedback," IEEE T. Syst. Rehabil. Eng., vol. 21, no. 1, pp. 112-120, 2013.

[128] A. J. Westerveld, A. C. Schouten, P. H. Veltink, and H. van der Kooij, "Selectivity and resolution of surface electrical stimulation for grasp and release," IEEE T. Syst. Rehabil. Eng., vol. 20, no. 1, pp. 94-101, 2012.

[129] S. Lim, D. Son, J. Kim, Y. B. Lee, J. Song, x, Kyul, S. Choi, D. J.
Lee, J. H. Kim, M. Lee, and T. Hyeon, "Transparent and stretchable interactive human machine interface based on patterned graphene heterostructures," Adv. Funct. Mater., vol. 25, no. 3, pp. 375-383, 2015.

[130] S. Dosen, M. Markovic, N. Wille, M. Henkel, M. Koppe, A. Ninu, C. Fr02mmel, and D. Farina, "Building an internal model of a myoelectric prosthesis via closed-loop control for consistent and routine grasping,” Exp. Brain Res., pp. 1-11, 2015.

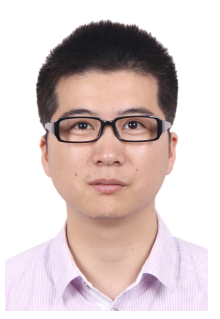

Yinfeng Fang (S'12) received the Ph.D. in Intelligent robotics from the University of Portsmouth, UK in 2015, and the B.S. degree in Electrical and Information Engineering and the M.S. degree in Pattern Recognition and Intelligent System from the Zhejiang University of Technology, China. Currently he is Research Fellow in the Intelligent System and Biomedical Robotics group at the University of Portsmouth, U.K.

His research interests are on human machine interface, bio-signal processing and machine learning.

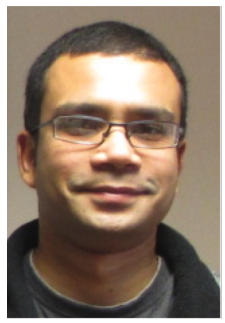

Nalinda Hettiarachchi received the BENG (Hons) in Automotive Engineering from Kingston University, London, UK, in 2006. He is currently working toward the $\mathrm{PhD}$ in Robotics at University of Portsmouth, Portsmouth, UK.

His research interests include Human Machine Interface, Signal Processing, Embedded systems and Mechatronics.

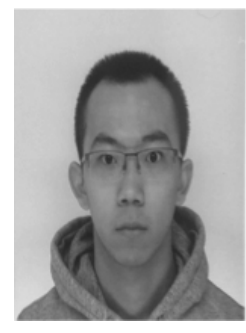

Dalin Zhou received the B.S. degree in Automation from University of Science and Technology of China in 2012, and is currently a Ph.D. candidate in the intelligent system and biomedical robotics group from the University of Portsmouth, Portsmouth, U.K. His research interests are signal processing and machine learning.

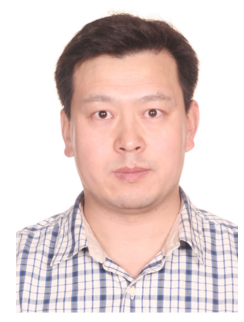

Honghai Liu (M02SM06) received the Ph.D. in intelligent robotics from Kings College London, London, U.K., in 2003. He is currently Chair Professor of intelligent systems and Robotics. His research interests include wearable sensing, biomechatronics, intelligent video analytics, intelligent robotics, and their applications with an emphasis on approaches that could make contribution to the intelligent connection of perception to action using contextual information.

$\mathrm{He}$ is Associate Editor of IEEE Transactions on Human Machine Systems, IEEE Transactions on Industrial Informatics and IEEE Transactions on Industrial Electronics. 